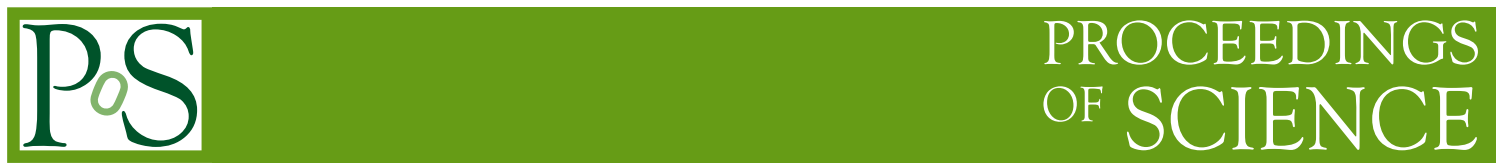

\title{
Alignment of the ALICE Tracking Detectors
}

\section{Andrea Dainese*, for the ALICE Collaboration}

INFN - Sezione di Padova, Padova, Italy

E-mail: andrea.dainese@pd.infn.it

\begin{abstract}
ALICE (A Large Ion Collider Experiment) is the LHC (Large Hadron Collider) experiment devoted to investigating the strongly interacting matter created in nucleus-nucleus collision at LHC energies. The ALICE Inner Tracking System (ITS) consists of six cylindrical layers of silicon detectors with three different technologies: pixels in the two innermost layers (SPD), drifts in the two intermediate layers (SDD), and strips in the two outer ones (SSD). The number of geometrical parameters to be determined in the ITS alignment is about 13,000 and the target precision is below $10 \mu \mathrm{m}$. The alignment procedure is intended to make use both of tracks from cosmic-ray muons and tracks from pp collisions. The main alignment method uses the Millepede approach, where a global fit to all residuals is performed, extracting all the misalignment parameters simultaneously. In this contribution we present the results obtained for the ITS alignment using about $10^{5}$ charged tracks from cosmic-rays that have been collected during summer 2008 with the ALICE magnetic field switched off.
\end{abstract}

VERTEX 2009 (18th workshop)

September 13-18 2009

VELUWE, the Netherlands

\footnotetext{
* Speaker.
} 

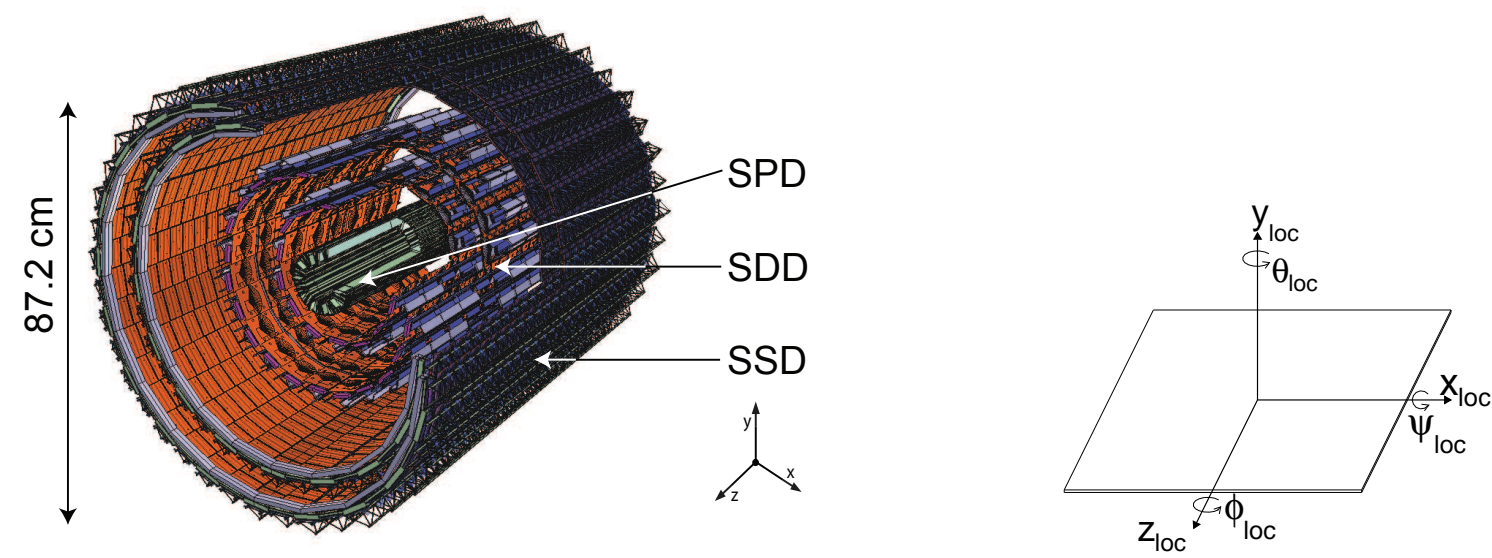

Figure 1: Layout of the ITS and definition of the ALICE global (left) and ITS-module local (right) reference systems.

\section{Introduction}

The ALICE Inner Tracking System (ITS) is a barrel-type silicon tracker that surrounds the interaction region [1]. It consists of six cylindrical layers, with radii between $3.9 \mathrm{~cm}$ and $43.0 \mathrm{~cm}$, covering the pseudo-rapidity range $|\eta|<0.9$. The two innermost layers are equipped with Silicon Pixel Detectors (SPD), the two intermediate layers are made of Silicon Drift Detectors (SDD), while Silicon Strip Detectors (SSD) are mounted on the two outermost layers. The main task of the ITS is to provide precise track and vertex reconstruction close to the interaction point. In particular, the ITS was designed with the aim to improve the position, angle, and momentum resolution for tracks reconstructed in the Time Projection Chamber (TPC), to identify the secondary vertices from the decay of hyperons and heavy flavoured hadrons, to reconstruct the interaction vertex with a resolution better than $100 \mu \mathrm{m}$, and to recover particles that are missed by the TPC (due to either dead regions or low-momentum cut-off). According to the design parameters, the position resolution at the primary vertex in the plane transverse to the beam-line for charged-pion tracks reconstructed in the TPC and in the ITS is expected to be approximately, in $\mu \mathrm{m}, 10+53 /\left(p_{\mathrm{t}} \sqrt{\sin \theta}\right)$, where $p_{\mathrm{t}}$ is the transverse momentum in $\mathrm{GeV} / c$ and $\theta$ is the polar angle with respect to the beam-line [2]. However, when considering the real detector, as installed in the experiment, the resolution is in general significantly degraded by misalignments. The ITS alignment procedure [3] starts from the positioning survey measurements performed during the assembly, and is refined using tracks from cosmic-ray muons and from particles produced in LHC pp collisions. Two independent methods, based on the minimization of tracks-to-measured-points residuals, are considered. The first method uses the Millepede approach [4], where a global fit to all residuals is performed, extracting all the alignment parameters simultaneously. The second method, not presented here, performs a (local) minimization for each single module and accounts for correlations between modules by iterating the procedure until convergence is reached [3].

\section{ITS detector parameters and alignment target}

The geometrical layout of the ITS layers, as it is implemented in the ALICE simulation and recon- 
struction software framework (AliRoot [5]), is shown in the left-hand panel of Fig. 1. The ALICE global reference system has the $z$ axis on the beam-line, the $x$ axis in the LHC (horizontal) plane, pointing to the centre of the accelerator, and the $y$ axis pointing upward. The axis of the ITS barrel coincides with the $z$ axis. The module local reference system (Fig. 1, right) is defined with the $x_{\text {loc }}$ and $z_{\text {loc }}$ axes on the sensor plane and the $z_{\text {loc }}$ axis in the same direction as the global $z$ axis. The local $x$ direction is approximately equivalent to the global $r \varphi$ at fixed $r$. The alignment degrees of freedom of the module are translations in $x_{\mathrm{loc}}, y_{\mathrm{loc}}, z_{\mathrm{loc}}$, and rotations by angles $\psi_{\mathrm{loc}}, \theta_{\mathrm{loc}}, \varphi_{\mathrm{loc}}$, about the $x_{\text {loc }}, y_{\text {loc }}, z_{\text {loc }}$ axes, respectively.

The geometrical parameters of the layers (radial position, length along beam axis, number of modules, spatial resolution, and material budget) are summarized in Table 1. In order to provide an acceptance coverage as hermetic as possible in the region $|\eta|<0.9$, in all six layer, the sensor modules are mounted at two slightly different radii or with a tilt (SPD outer layer) so as to have small acceptance overlaps of the order of $2 \%$ in both the $x_{\text {loc }}$ and $z$ directions. These overlaps (which are lacking only for SPD in the $z$ direction) are extremely useful to evaluate the quality of the alignment, as we will detail in the following sections.

In the SDD detectors, while the $z$ coordinate is reconstructed from the centroid of the collected charge along the anodes, the position along the drift coordinate $\left(x_{\mathrm{loc}} \approx r \varphi\right)$ is reconstructed starting from the measured drift time with respect to the trigger time. An unbiased reconstruction of the $x_{\text {loc }}$ coordinate requires therefore to know with good precision the drift speed and the time-zero $\left(t_{0}\right)$, which is the measured drift time for particles with zero drift distance. For this reason, the calibration and alignment procedures for the SDD are closely related.

The target of the alignment procedures is the achievement of a level of precision and accuracy such that the resolution on the reconstructed track parameters (in particular, the impact parameter and the curvature, which measures the transverse momentum) is degraded by at most $20 \%$ with respect to the resolution expected in case of the ideal geometry without misalignment. With reference to the intrinsic precisions listed in Table 1, the target residual misalignment spreads in the local coordinates on the sensor plane are: for SPD, $8 \mu \mathrm{m}$ in $x_{\mathrm{loc}}$ and $70 \mu \mathrm{m}$ in $z_{\text {loc }}$; for SDD, $25 \mu \mathrm{m}$ in $x_{\mathrm{loc}}$ and $18 \mu \mathrm{m}$ in $z_{\mathrm{loc}}$; for SSD, $14 \mu \mathrm{m}$ in $x_{\mathrm{loc}}$ and $500 \mu \mathrm{m}$ in $z_{\mathrm{loc}}$. Since also the misalignment in the $\theta_{\text {loc }}$ angle (rotation about the axis normal to the sensor plane) impacts directly on the effective spatial precision, the numbers given above should be taken as effective spreads including also the effect of the $\theta_{\text {loc }}$ rotation. In any case, these target numbers are only an indication of the precision

Table 1: Characteristics of the six ITS layers.

\begin{tabular}{|c|c|c|c|c|c|c|c|}
\hline Layer & Type & $r[\mathrm{~cm}]$ & $\pm z[\mathrm{~cm}]$ & $\begin{array}{c}\text { Number } \\
\text { of } \\
\text { modules }\end{array}$ & $\begin{array}{c}\text { Active Area } \\
\text { per module } \\
x_{\text {loc }} \times z\left[\mathrm{~mm}^{2}\right]\end{array}$ & $\begin{array}{c}\text { Material } \\
x_{\text {loc }} \times z_{\text {loc }}\left[\mu \mathrm{m}^{2}\right]\end{array}$ & $\begin{array}{c}X / X_{0}[\%] \\
\text { budget }\end{array}$ \\
\hline \hline 1 & pixel & 3.9 & 14.1 & 80 & $12.8 \times 70.7$ & $12 \times 100$ & 1.14 \\
2 & pixel & 7.6 & 14.1 & 160 & $12.8 \times 70.7$ & $12 \times 100$ & 1.14 \\
3 & drift & 15.0 & 22.2 & 84 & $70.17 \times 75.26$ & $35 \times 25$ & 1.13 \\
4 & drift & 23.9 & 29.7 & 176 & $70.17 \times 75.26$ & $35 \times 25$ & 1.26 \\
5 & strip & 38.0 & 43.1 & 748 & $73 \times 40$ & $20 \times 830$ & 0.83 \\
6 & strip & 43.0 & 48.9 & 950 & $73 \times 40$ & $20 \times 830$ & 0.86 \\
\hline
\end{tabular}


that is requested from the alignment procedures.

\section{Cosmic-ray run 2008: data taking and reconstruction}

During the 2008 cosmic run, extending from June to October, about $10^{5}$ events with reconstructed tracks in the ITS have been collected with the magnetic field switched off, using the FastOR trigger provided by the Silicon Pixel Detector. The SPD FastOR trigger [1] is based on a programmable hit pattern recognition system (on FPGA) at the level of individual readout chips (1200 in total, each reading a sensor area of about $1.4 \times 1.4 \mathrm{~cm}^{2}$ ). For the 2008 cosmic run, the trigger logic consisted in selecting events with at least one hit on the upper half of the outer SPD layer $(r \approx 7 \mathrm{~cm})$ and at least one on the lower half of the same layer. For the FastOR trigger, typically $77 \%$ of the chips (i.e. about $90 \%$ of the active modules) could be configured and used, and the typical trigger rate was about $0.18 \mathrm{~Hz}$.

The following procedure, fully integrated in the AliRoot framework [5], is used for track reconstruction: after the cluster finding in the ITS (hereafter, we will refer to the clusters as "points"), a pseudo primary vertex is created using the reconstructed points in the two SPD layers; track reconstruction is then performed using the ITS standalone tracker, which finds tracks in the outward direction, from the innermost SPD layer to the outermost SSD layer. During the final track refit stage, when the already identified ITS points are used in the Kalman-filter fit in the inward direction, in order to obtain the track parameters estimate at the (pseudo) vertex, "extra" points are searched for in the ITS module overlaps. Currently, the "extra" points are not used to update the track parameters, so they can be exploited as a powerful tool to evaluate the ITS alignment quality.

The main limitation of the usage of cosmic-ray tracks for the alignment of a cylindrical detector like the ITS is that the occupancy of the side modules is small, especially for the external layers. This is due to the small size of the triggering detector (SPD), the dominance of small zenith angles for cosmic-ray particles and the cut on the track-to-module incidence angle $\left(>30^{\circ}\right)$ that we apply to reject large and elongated clusters. On the other hand, cosmic-ray tracks offer a powerful constraint against the so-called "weak modes", correlated misalignments that are difficult to determine with radial tracks produced in collisions, like radial layer deformations and top-bottom relative shifts.

\section{Validation of the SSD survey measurements}

The SDD and SSD were surveyed during the assembling phase using a measuring machine with an intrinsic resolution of about $5-10 \mu \mathrm{m}$ in each coordinate. The survey, very similar for the two detectors, was carried out in two stages: the measurement of the positions of the modules on the ladders and the measurement of the positions of the ladder endpoints on the support cone. The typical magnitude of the survey measurements is $\approx 20-50 \mu \mathrm{m}$. The validation of the SSD survey measurements with cosmic-ray data was performed with three independent methods [6].

The first method uses the double points in acceptance overlaps, which allow us to estimate the effective spatial resolution of the sensor modules. We define the distance $\Delta x_{\text {loc }}$ between the two points in the local $x$ direction on the module plane by projecting the point of one of the two modules on the other module plane, along the track direction. Figure 2 shows the $\Delta x_{\text {loc }}$ distributions 

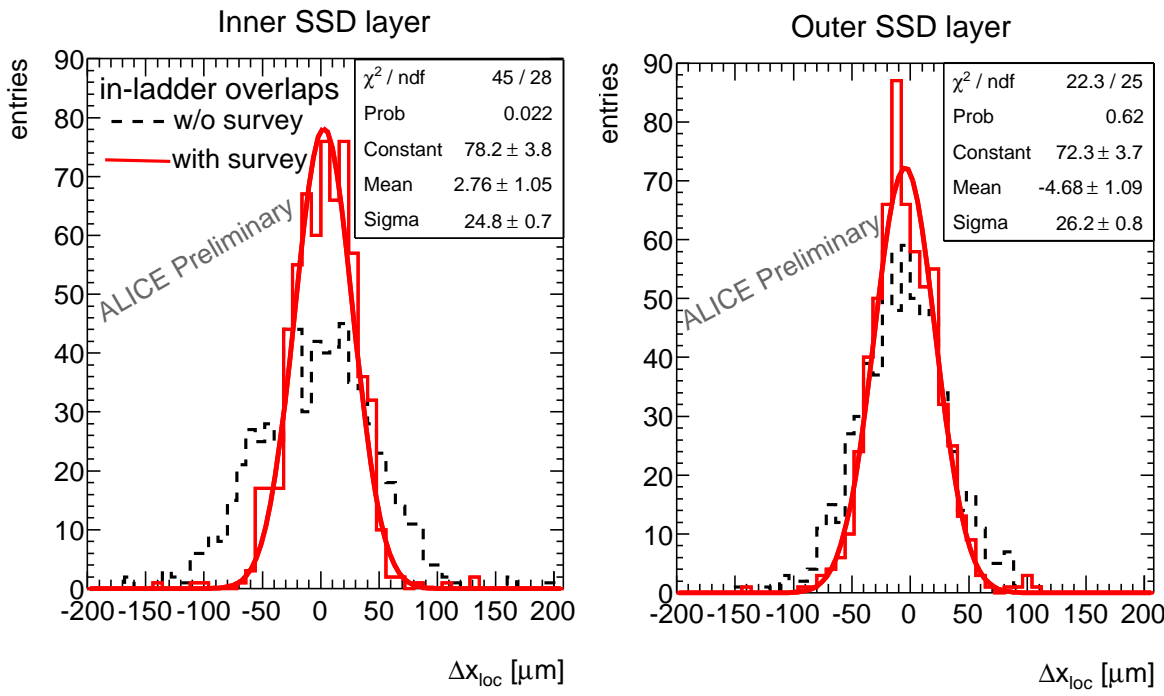

Figure 2: Distribution of $\Delta x_{\text {loc }}$, the distance between two points in the module overlap regions along $z$ on the same ladder, for the two layers of the SSD.

without and with the survey corrections, for the two SSD layers. When the survey corrections are applied, the spread of the distributions, obtained from a gaussian fit, is $\sigma \approx 25.5 \mu \mathrm{m}$. This arises from the combined spreads of the two points, thus the effective position resolution for a single point is estimated to be smaller by a factor $1 / \sqrt{2}$, i.e. $\approx 18 \mu \mathrm{m}$, which is compatible with the expected intrinsic spatial resolution of about $20 \mu \mathrm{m}$. This indicates that the residual misalignment after applying the survey is compatible with the expected precision of the survey measurements of $\approx 5 \mu \mathrm{m}$.

Another test that was performed uses two points in the outer SSD layer to define a straight track (no magnetic field) and inspects the residuals between points on the inner layer and the track. The point on the inner layer is required to be between the two points on the the outer layer. The effective resolution of the points in $z$ was found to be about $780 \mu \mathrm{m}$, indicating that no significant additional misalignment is present. For the $x_{\text {loc }}$ direction, the obtained spread of $25 \mu \mathrm{m}$ is larger than the intrinsic resolution of $20 \mu \mathrm{m}$. Multiple scattering of low-momentum tracks is expected to contribute to the broadening of the distribution, but no quantitative estimate of this effect was carried out. We can therefore not rule out that additional misalignments with a Gaussian sigma up to about $20 \mu \mathrm{m}$ are present in the SSD. The mean residual is also non-zero, $(3.9 \pm 0.4) \mu \mathrm{m}$, which suggests that residual shifts at the 5-10 $\mu \mathrm{m}$ level could be present. These misalignments would have to be at the ladder level to be compatible with the result from the study with sensor module overlaps.

A third method that was used to verify the SSD survey consisted of performing tracking with pairs of points ( 2 points on the inner and two points on the outer layer or two sets of points on each layer), and comparing the track parameters of both track segments. The conclusion from this method is consistent with the results from the track-to-point method [6]. 


\section{ITS alignment with Millepede}

In general, the task of track-based alignment algorithms is the determination of the set of geometry parameters that minimize the global $\chi^{2}$ of the track-to-point residuals:

$$
\chi_{\text {global }}^{2}=\sum_{\text {modules, tracks }} \vec{\delta}_{t, p}^{\mathrm{T}} \mathbf{V}_{t, p}^{-1} \vec{\delta}_{t, p}
$$

In this expression, the sum runs over all the detector modules and all the tracks in a given dataset; $\vec{\delta}_{t, p}=\vec{r}_{t}-\vec{r}_{p}$ is the residual between the data point $\vec{r}_{p}$ and the reconstructed track extrapolation $\vec{r}_{t}$ to the module plane; $\mathbf{V}_{t, p}$ is the covariance matrix of the residual.

Millepede $[4,7]$ — the main algorithm used for ITS alignment — belongs to the global leastsquares minimization type of algorithms, which aim at determining simultaneously all the parameters that minimize the global $\chi^{2}$ in Eq. (5.1). The idea behind the Millepede method is to consider the local parameters (track parameters) as nuisance parameters and to compute explicitly only the global parameters (alignment parameters). In our implementation of the Millepede algorithm, we paid special attention to the possibility to account for the complex hierarchy of the alignable volumes of the ITS, in general leading to better description of the material budget distribution after alignment. This is achieved by defining explicit parent-daughter relationships between the volumes corresponding to mechanical degrees of freedom in the ITS.

The SPD detector was first aligned using about $5 \times 10^{4}$ cosmic-ray tracks, with two points in the inner layer and two points in the outer layer, collected in 2008 with the magnetic field switched off. A hierarchical alignment procedure was adopted, starting from largest structures (the ten sectors that form the SPD cylinder) and ending with the single sensor modules.

Mainly, the following two observables are used to check the quality of the obtained alignment: the top half-track to bottom half-track matching at the plane $y=0$, and the track-to-point distance for the "extra" points in the acceptance overlaps.

For the first observable, the main variable is $\left.\Delta x y\right|_{y=0}$, the track-to-track distance at $y=0$ in the $(x, y)$ plane transverse to the beam-line. This observable, that is accessible only with cosmic-ray tracks, provides a direct measurement of the resolution on the track transverse impact parameter $d_{0}$; namely: $\sigma_{\Delta x y \mid y=0}\left(p_{\mathrm{t}}\right)=\sqrt{2} \sigma_{d_{0}}\left(p_{\mathrm{t}}\right)$. Since the data used for the current analysis were collected without magnetic field, they do not allow us to directly assess the $d_{0}$ resolution. However, also without a momentum measurement, $\left.\Delta x y\right|_{y=0}$ is a powerful indicator of the alignment quality, as we show in the following.

Figure 3 (left) shows the distribution of $\left.\Delta x y\right|_{y=0}$ for SPD. The two track segments are required to have a point in each of the SPD layers and to pass, within $1 \mathrm{~cm}$ from the origin in the transverse plane (this cut selects tracks with a similar topology as those produced in collisions and, in particular, rejects tracks that have small incidence angles on the inner layer modules). A gaussian fit to the final distribution in the range $[-100 \mu \mathrm{m},+100 \mu \mathrm{m}]$, gives a centroid compatible with zero and a spread $\sigma \approx 50 \mu \mathrm{m}$. For comparison, a spread of $38 \mu \mathrm{m}$ is obtained from a Monte Carlo simulation of comic-muons generated according to the momentum spectrum measured by the ALICE TPC in cosmic runs with magnetic field, with the ideal geometry of the ITS (without misalignment). When only the SPD detector is used and the tracks are straight lines (no magnetic field), the spread of the $\left.\Delta x y\right|_{y=0}$ distribution can be related in a simple way to the effective spatial resolution $\sigma_{\text {spatial }}$, 

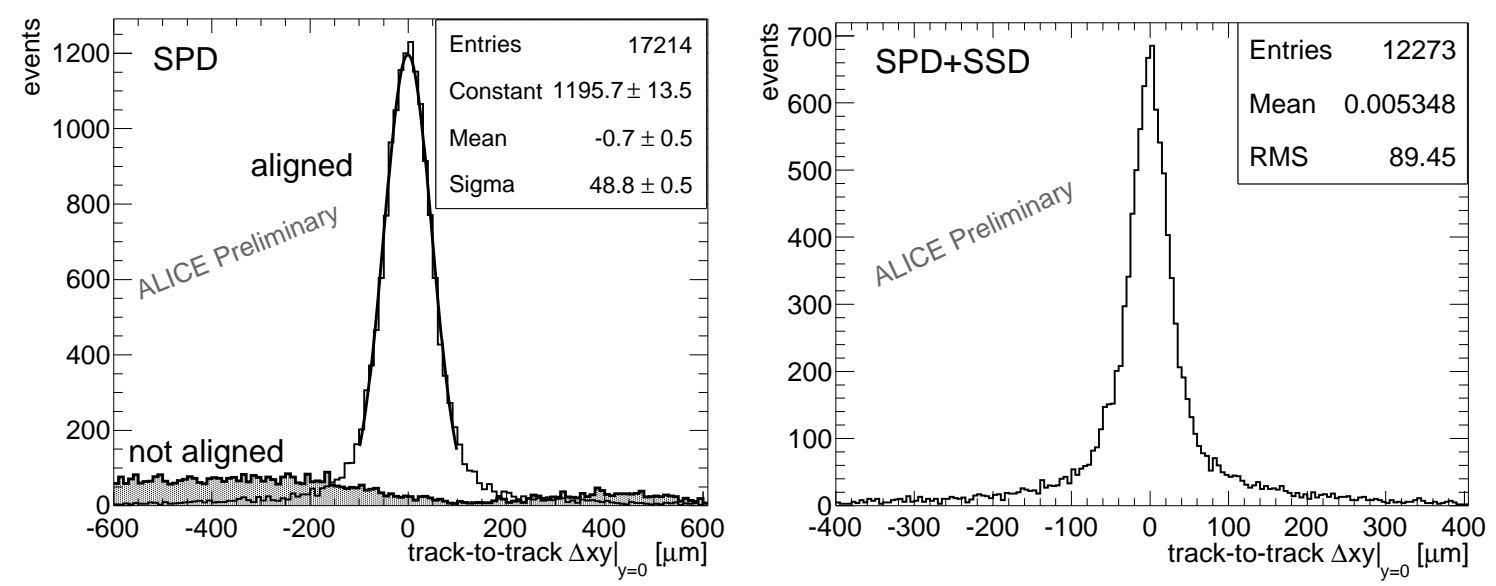

Figure 3: Left: distribution of $\left.\Delta x y\right|_{y=0}$ for SPD, without alignment and with the Millepede alignment corrections. Right: distribution of $\left.\Delta x y\right|_{y=0}$ for SPD+SSD, with the Millepede alignment corrections.

inclusive of the intrinsic sensor resolution and of the residual misalignment. For tracks passing close to the beam line (as in our case, with the cut at $1 \mathrm{~cm}$ ), we have:

$$
\sigma_{\left.\Delta x y\right|_{y=0}}^{2} \approx 2 \frac{\left(r_{\mathrm{SPD} 1}^{2} \sigma_{\mathrm{spatia}, \mathrm{SPD} 1}^{2}+r_{\mathrm{SPD} 2}^{2} \sigma_{\mathrm{spatial}, \mathrm{SPD} 2}^{2}\right)}{\left(r_{\mathrm{SPD} 1}-r_{\mathrm{SPD} 2}\right)^{2}} \approx 2 \frac{r_{\mathrm{SPD} 1}^{2}+r_{\mathrm{SPD} 2}^{2}}{\left(r_{\mathrm{SPD} 1}-r_{\mathrm{SPD} 2}\right)^{2}} \sigma_{\mathrm{spatial}}^{2},
$$

where the inner and outer SPD layers are indicated as SPD1 and SPD2, respectively. This relation neglects the effect of multiple scattering in the pixels and in the beam pipe, which is certainly one of the reasons why the $\left.\Delta x y\right|_{y=0}$ distribution is not gaussian outside the central region, most likely populated by the high-momentum component of the cosmic muons. Using the fit result, $\sigma_{\left.\Delta x y\right|_{y=0}} \approx 50 \mu \mathrm{m}$, obtained in the central region $[-100 \mu \mathrm{m},+100 \mu \mathrm{m}]$, we estimate the value $\sigma_{\text {spatial }} \approx 14 \mu \mathrm{m}$, not far from the intrinsic resolution of about $11 \mu \mathrm{m}$ extracted from the simulation.

The next step in the alignment procedure is the inclusion of the SSD detector. As shown in section 4 , the survey measurements already provide a very precise alignment, with residual misalignment levels of the order of less than $5 \mu \mathrm{m}$ for modules on the ladder and of about $20 \mu \mathrm{m}$ for ladders. Because of the limited available statistics $\left(\approx 2 \times 10^{4}\right.$ tracks with four points in SPD and four points in SSD), the expected level of alignment obtained with Millepede on single SSD modules is significantly worse than the level reached with the survey measurements. For this reason, Millepede was used only to align the whole SPD barrel with respect to the SSD barrel and to optimize the positioning of large sets of SSD modules, namely the upper and lower halves of layers 5 and 6. Figure 3 (right) shows the distribution of $\left.\Delta x y\right|_{y=0}$ for pairs of track segments, each reconstructed with two points in SPD and two in SSD, i.e. the merged cosmic-ray track has eight points in SPD+SSD. It can be seen that, when the SSD survey and the Millepede alignment are applied, the distribution is centred at zero and very narrow (FWHM $\approx 60 \mu \mathrm{m}$ ), but it shows nongaussian tails, most likely due to multiple scattering. A more precise alignment of the SSD using high-momentum tracks will be performed with the 2009 cosmic-ray and proton-proton data.

The second alignment quality observable is the $\Delta x_{\text {loc }}$ distance between points in the region where there is an acceptance overlap between two modules of the same layer. In Fig. 4 (left), we show the track-to-point distance $\Delta x_{\text {loc }}$ for the SPD "extra" points in the transverse plane, before 

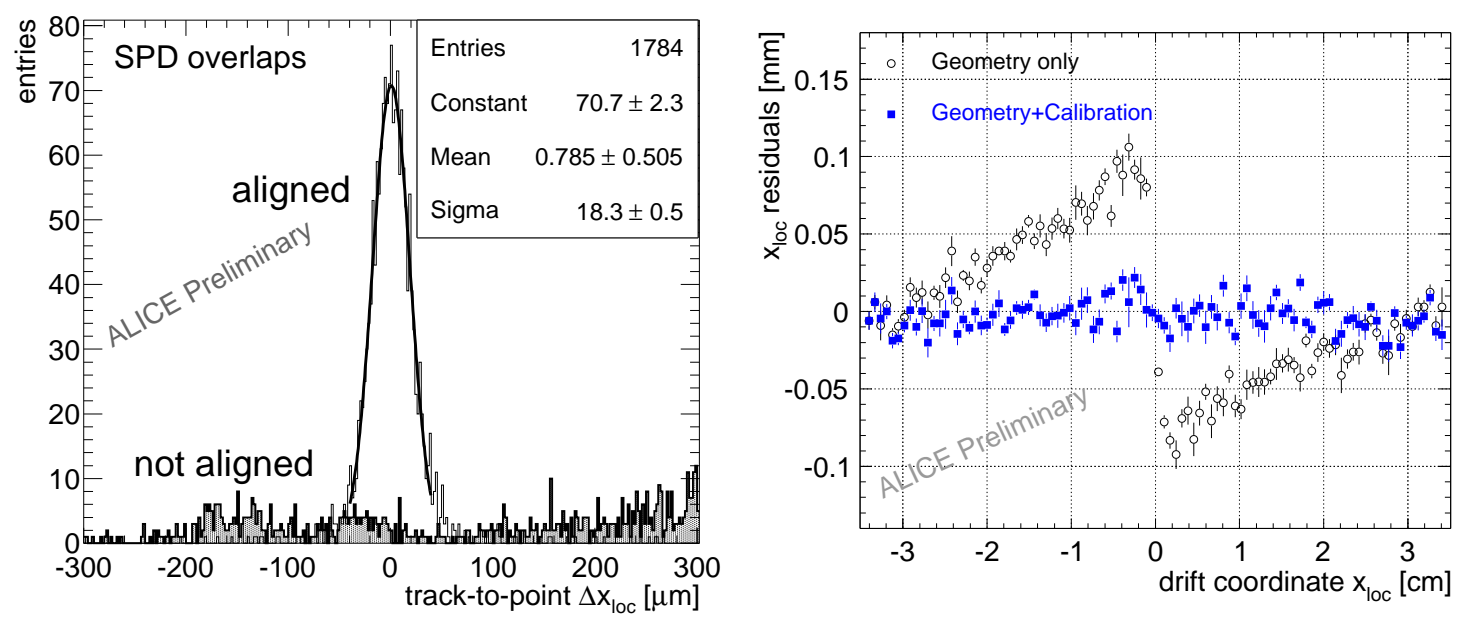

Figure 4: Left: SPD track-to-point $\Delta x_{\text {loc }}$ for "extra" points in acceptance overlaps before and after alignment. Right: SDD residuals along the drift coordinate for one module as a function of drift coordinate after Millepede alignment with only geometrical parameters and with geometrical+calibration parameters.

and after the Millepede alignment. The extra points are not used in the alignment procedure. The spread of the distribution is $\sigma \approx 18 \mu \mathrm{m}$, which indicates a single point effective resolution $\sigma_{\text {spatial }} \approx 13 \mu \mathrm{m}$, to be compared to the value of about $11 \mu \mathrm{m}$ obtained from the simulation.

The alignment of the SDD detectors for the $x_{\text {loc }}$ coordinate (reconstructed from the drift time) is complicated by the interplay between the geometrical misalignment and the calibration of drift speed and $t_{0}$. After a first calibration with SDD-standalone methods, a refinement of the determination of these parameters can obtained within the Millepede approach by adding them as free global parameters for each of the 260 SDD modules. This allows to assess at the same time geometrical alignment and calibration parameters of the SDD detectors. An example is shown for a specific SDD module in Fig. 4 (right), where the $x_{\text {loc }}$ residuals along the drift direction are shown as a function of $x_{\mathrm{loc}}$. The clear systematic shift between the two drift regions $\left(x_{\mathrm{loc}}<0\right.$ and $\left.x_{\mathrm{loc}}>0\right)$, visible when only the geometrical parameters are included in Millepede, is due to both mis-calibrated $t_{0}$ and biased drift speed (this is a module with non-working injectors). These systematic effects are no longer present when also the calibration parameters are fitted by Millepede.

\section{Conclusions}

The results on the first alignment of the ALICE Inner Tracking System with cosmic-ray tracks, collected in 2008 in the absence of magnetic field, have been presented. More details can be found in Ref. [3]. The obtained alignment corrections can be reliably applied to the first pp data with magnetic field on, because the ALICE barrel field is relatively weak $(0.5 \mathrm{~T})$ and we do not expect it to determine geometrical deformations of the ITS.

The initial step of the alignment procedure consisted in the validation of the survey measurements for the Silicon Strip Detector (SSD), which indicates that the residual misalignment for modules on ladders is within $5 \mu \mathrm{m}$, while the residual misalignment for the ladders with respect to the support cones amounts to about $20 \mu \mathrm{m}$. 
The procedure continues with track-based software alignment performing residuals minimization. We mainly use the Millepede algorithm, which minimizes a global $\chi^{2}$ of residuals for all alignable volumes and a large set of tracks. We start from the SPD, which is aligned in a hierarchical approach. Then, we align the SPD barrel with respect to the SSD barrel. The two intermediate ITS layers, the Silicon Drift Detectors (SDD), represent a special case, because the reconstruction of one of the two local coordinates requires dedicated calibration procedures (drift velocity and drift time zero extraction), which are to some extent related to the alignment. Indeed, one of the approaches that we are developing for the time zero calibration is based on the analysis of track residuals in a standalone procedure, initially, and then directly within the Millepede algorithm. Once these procedures will become stable and robust, the SDD will be included in the standard alignment chain.

We use mainly two observables to assess the quality of the obtained alignment: the matching of the two half-tracks produced by a cosmic-ray particle in the upper and lower halves of the ITS barrel, and the residuals between double points produced in the geometrical overlaps between adjacent modules. For the SPD, both observables indicate an effective space point resolution of about $14 \mu \mathrm{m}$ in the most precise direction, to be compared to about $11 \mu \mathrm{m}$ extracted from the Monte Carlo simulation without misalignments. This difference of $\approx 25 \%$ (from 11 to $14 \mu \mathrm{m}$ ) is already rather close the $20 \%$, which is the final target of the alignment. Further confidence on the robustness of the results is provided by the comparison of the Millepede results to those from a second, independent, alignment method. This second method, which iteratively minimizes a set of local module-by-module $\chi^{2}$ functions, yields, compared to Millepede, a similar alignment quality and a compatible set of alignment corrections.

For all six layers, the completion of the alignment for all modules will require tracks from proton-proton collisions; a few $10^{6}$ events should allow us to reach a uniform alignment level, close to the target, over the entire detector.

\section{References}

[1] K. Aamodt et al. [ALICE Collaboration], JINST 3 (2008) S08002.

[2] B. Alessandro et al. [ALICE Collaboration], J. Phys. G 32 (2006) 1295.

[3] C. Bombonati et al., ALICE Internal Note 2009-035 (2009).

[4] V. Blobel and C. Kleinwort, contribution to the Conference on Advanced Statistical Techniques in Particle Physics, Durham, March, 18-22, 2002.

[5] ALICE Off-line framework, AliRoot, http://aliceinfo.cern.ch/Offline

[6] A. Dainese et al., ALICE Internal Note 2009-045 (2009).

[7] V. Blobel, in "Proceedings of the first LHC detector alignment workshop", CERN, September 4-6, 2006, CERN-2007-004. 